\title{
The Performance Merit of Dynamic Threshold Energy Detection Algorithm in Cognitive Radio Systems
}

\author{
Jinbo Wu, Guicai Yu, Tao Luo and Guangxin Yue \\ school of Information and Communication Engineering, \\ Beijing University of Posts and Telecommunications, \\ Beijing, P.R. China 100876. \\ E-mail:hyxwu@163.com, \{tluo,gxyue\}@bupt.edu.cn
}

\begin{abstract}
In cognitive radio systems, secondary users should determine correctly whether the primary user is absent or not in a certain spectrum within a short detection period. Traditional spectrum sensing schemes based on fixed threshold are sensitive to noise uncertainty, a fractional fluctuate of average noise power in a short time will lead to the accuracy of spectrum detection decreasing seriously. In this paper we propose a novel dynamic threshold energy detection algorithm in cognitive radio systems. Theoretical analysis and simulation results show that the proposed strategy can combat the noise uncertainty effectively, and good detection accuracy can be attained, if a suitable detection threshold is chosen. That is, the proposed scheme can enhance the robustness of combatting the noise uncertainty and veracities of the spectrum sensing.
\end{abstract}

Index Terms-cognitive radio; detection threshold; noise uncertainty; dynamic threshold detection

\section{INTRODUCTION}

Nowadays wireless communication systems are based basically on fixed spectrum allocations, which means that the spectral is assigned fixedly to authorized users at any time. This leads to that the spectral resource is utilized un-efficiently through practical measurements [1]. Dynamic spectrum access techniques increase the spectrum efficiency and enhance the chance of secondary users to access the idle frequency spectrum. Cognitive radio is a resource sharing strategy which allows the licensed user to share parts of its licensed spectrum with a rental system (RS)[2], and the rental users must vacate the spectrum as long as licensed users need communicate. The objective of the cognitive radio is to improve the spectral efficiency by overlaying new wireless radio systems on a licensed one without interfering to the licensed system (LS). In order to keep co-existing and no harmful to LS, cognitive nodes must have the capability to detect the idle spectrum instantaneously and continually, which is a very important in cognitive radio systems.

The essential problem of spectrum sensing in cognitive radio is designing high quality spectrum sensing devices and algorithms for exchanging spectrum sensing data between nodes. Energy detection is a respected spectrum sensing scheme. Most papers[3] [8] discussed energy detection scheme based on a given invariant average noise power. However, the noise is an aggregation of various sources like thermal noise, leakage of signals, aliasing from imperfect front end filters, quantization noise, etc. Actually, "noise" is neither perfectly Gaussian nor white, nor stationary[9]. Therefore, it is not practical that the average noise power keeps constant in detection duration, In fact, the noise uncertainty is unavoidable. It has been shown that a simple energy detector cannot guarantee the accurate of signal detection, especially when the noise is uncertain. Hence, the conventional energy detection scheme is sensitive to noise uncertainty. For those reason, a novel energy detection algorithm based on dynamic threshold is presented to deal with the noise uncertainty in this paper.

The rest of this paper is organized as follows: In section II, the signal detection model in AWGN is formulated. Then, a noise uncertainty effects on the performance of energy detector is evaluated in section III. A novel algorithm is proposed, which applies dynamic threshold for anti-noise uncertainty in section IV. Conclusions are drawn in section V.

\section{Detection Model}

In this paper, the signal is assumed to be independent of the noise. Random processes are also assumed to be stationary and ergodic unless specified. The problem of signal detection in additive Gaussian noise can be formulated as a binary hypothesis testing problem with the following hypotheses

$$
\begin{cases}\mathcal{H}_{0}: Y(n)=W(n) & , n=1,2, \ldots, N \\ \mathcal{H}_{1}: Y(n)=X(n)+W(n) & , n=1,2, \ldots, N\end{cases}
$$

where $Y(n), X(n)$ and $W(n)$ are the received signals at $\mathrm{CR}$ nodes, transmitted signals at primary nodes and white noise samples, respectively; $\mathcal{H}_{0}$ and $\mathcal{H}_{1}$ denote that the licensed user is present or not, respectively. Noise samples $W(n)$ are from AWGN process with power spectral density $\sigma_{n}^{2}$, i.e., $W(n) \sim$ $\mathcal{N}\left(0, \sigma_{n}^{2}\right)$.

If there is no deterministic knowledge about the signal $X(n)$, i.e., we only know the average power of the signal. In this case the optimal detector is energy detector or radiometer [10], the test statistic is given by 


$$
D(Y)=\frac{1}{N} \sum_{n=0}^{N-1} Y^{2}(n) \stackrel{\mathcal{H}_{1}}{\underset{\mathcal{H}_{0}}{\gtrless}} \gamma
$$

where $D(Y)$ is the decision variable and $\gamma$ is the decision threshold, $N$ is the number of samples. If the noise variance is known and without noise uncertainty, based on central limit theorem(CLT), it has: [9][11]

$$
\left\{\begin{array}{l}
D\left(Y \mid \mathcal{H}_{0}\right) \sim \mathcal{N}\left(\sigma_{n}^{2}, 2 \sigma_{n}^{4} / N\right) \\
D\left(Y \mid \mathcal{H}_{1}\right) \sim \mathcal{N}\left(P+\sigma_{n}^{2}, 2\left(P+\sigma_{n}^{2}\right)^{2} / N\right)
\end{array}\right.
$$

where $P=\frac{1}{N}\left(\sum_{n=1}^{N}|X(n)|^{2}\right)$ is the average signal power, $\sigma_{n}^{2}$ is the noise variance. Then, we can obtain the probability of detection, false alarm probability, and missing probability, respectively [9][11].

$$
\begin{gathered}
P_{D}=\operatorname{Pr}\left(D(Y)>\gamma \mid \mathcal{H}_{1}\right)=Q\left(\frac{\gamma-\left(P+\sigma_{n}^{2}\right)}{\sqrt{2 / N}\left(P+\sigma_{n}^{2}\right)}\right) \\
P_{F A}=\operatorname{Pr}\left(D(Y)>\gamma \mid \mathcal{H}_{0}\right)=Q\left(\frac{\gamma-\sigma_{n}^{2}}{\sqrt{2 / N} \sigma_{n}^{2}}\right) \\
P_{M D}=1-P_{D}=1-Q\left(\frac{\gamma-\left(P+\sigma_{n}^{2}\right)}{\sqrt{2 / N}\left(P+\sigma_{n}^{2}\right)}\right)
\end{gathered}
$$

where $Q(\cdot)$ is the standard Gaussian complementary cumulative distribution function $(C D F) . P_{D}, P_{F A}$ and $P_{M D}$ represent detection probability, false alarm probability and missing probability respectively.

\section{Problem Formulation}

Given target variables: detection probability $P_{D}$, false alarm probability $P_{F A}$, missing probability $P_{M D}$ and sample number $N$ (detection duration), the relationship of these variables is deduced in terms of the signal-to-noise ratio $(S N R)$

To simplify the problem, energy detection algorithm based on average noise power without uncertainty has been discussed. From section II, eliminating the variable of decision threshold $\gamma$, and it has

$$
N=2\left[Q^{-1}\left(P_{F A}\right)-Q^{-1}\left(P_{D}\right)(1+S N R)\right]^{2} S N R^{-2}
$$

where $Q^{-1}(\cdot)$ is the inverse standard Gaussian complementary cumulative distribution function $(C D F), S N R=P / \sigma_{n}^{2}$.

Fig. 1 is the numerical results of (7) given $S N R=-10 \mathrm{~dB}$, $P_{F A} \in(0,0.5)$. It shows that the performance is improved gradually with $N$ 's increasing, and an accurate detection probability can be obtained even if the $S N R$ is much lower, as long as $N$ is large enough without noise uncertainty.

We have discussed the detection performance without noise uncertainty. Next, we take the noise uncertainty into account. In order to discuss the effect on the detection performance as the noise power is uncertain, we set: $\sigma^{2} \in\left[\sigma_{n}^{2} / \rho, \rho \sigma_{n}^{2}\right]$, where $\rho$ is the noise uncertainty factor and the value of $\rho$ is closer to 1 , that is $\rho>1$ and $\rho \approx 1$. Thus (4) and (5) are modified to get

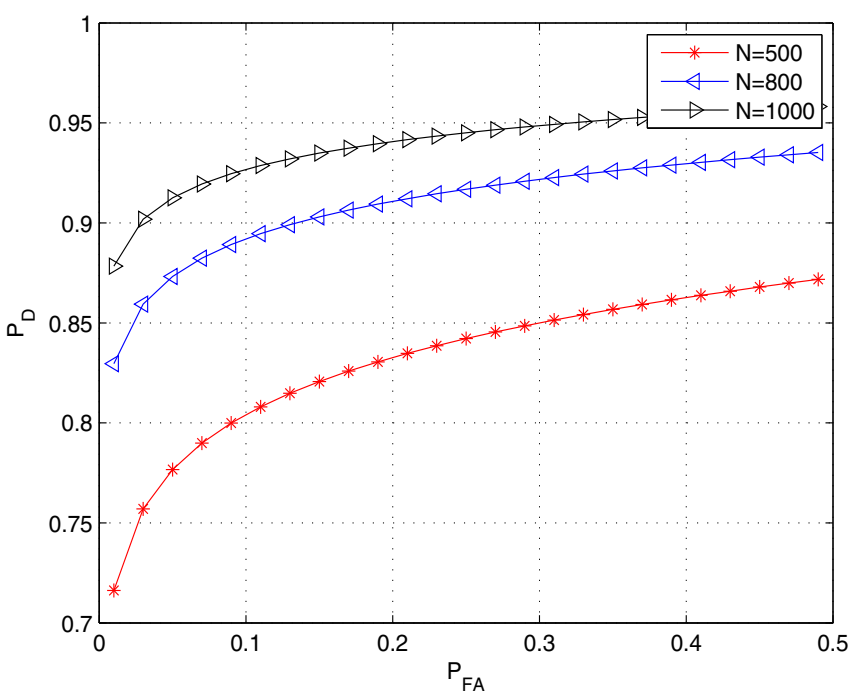

Fig. 1. ROC curves of energy detection scheme with different $N$

$$
\begin{gathered}
P_{D}=\min _{\sigma^{2} \in\left[\sigma_{n}^{2} / \rho, \rho \sigma_{n}^{2}\right]} Q\left(\frac{\gamma-\left(P+\sigma^{2}\right)}{\sqrt{2 / N}\left(P+\sigma^{2}\right)}\right) \\
=Q\left(\frac{\gamma-\left(P+\sigma_{n}^{2} / \rho\right)}{\sqrt{2 / N}\left(P+\sigma_{n}^{2} / \rho\right)}\right) \\
P_{F A}=\max _{\sigma^{2} \in\left[\sigma_{n}^{2} / \rho, \rho \sigma_{n}^{2}\right]} Q\left(\frac{\gamma-\sigma^{2}}{\sqrt{2 / N} \sigma^{2}}\right)=Q\left(\frac{\gamma-\rho \sigma_{n}^{2}}{\sqrt{2 / N} \rho \sigma_{n}^{2}}\right)
\end{gathered}
$$

Eliminating $\gamma$,and it has:

$$
\begin{aligned}
N & =2\left[\rho Q^{-1}\left(P_{F A}\right)-(1 / \rho+S N R) Q^{-1}\left(P_{D}\right)\right]^{2} \\
& \times(S N R-(\rho-1 / \rho))^{-2}
\end{aligned}
$$

Comparing (10) with (7), there is almost no contribution to the whole expression results if there is a tiny change of $\rho$; however, $S N R^{-2}$ and $(S N R-(\rho-1 / \rho))^{-2}$ should be mainly discussed and compared. When $\rho \approx 1$, then $S N R^{-2} \approx$ $(S N R-(\rho-1 / \rho))^{-2}$, the numerical value of (10) and (7) are almost the same; When $\rho$ is larger and suppose $\rho=1.05$, then $(\rho-1 / \rho)=0.0976 \approx 0.1$, if $S N R=0.1$, well then $(S N R-(\rho-1 / \rho))^{-2} \approx 0$, substituting into equation (10) to be $N \rightarrow \infty$. In other words, only an infinite detection duration can complete detection, which is impracticable. A tiny fluctuation of average noise power causes performance drop seriously, especially with a lower $S N R$.

Fig.2 is the numerical results of (10) given: $S N R=-10 \mathrm{~dB}$, $P_{F A} \in(0,0.5)$ and $N=1000$.

In Fig.2, $\rho=1.00$ represents no noise uncertainty, namely the average noise power keeps constant in short time. We can see that the performance gradually drops as the noise uncertainty factor increasing. When $\rho=1.05$, the performance dropped seriously. For example, if $P_{F A}=0.1$, then $P_{D}<0.30$, even when $P_{F A}=0.5$, the detection probability is still less 


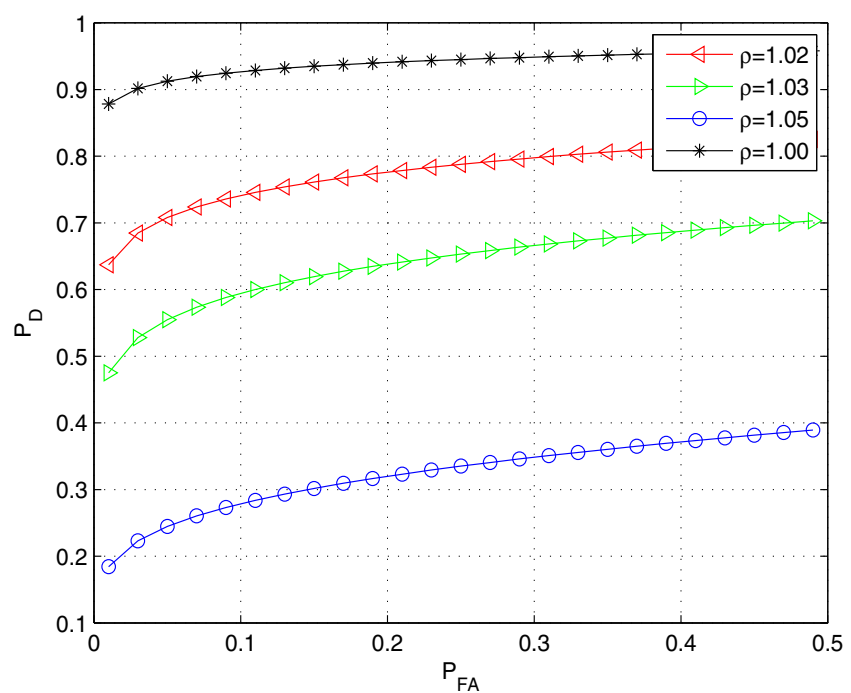

Fig. 2. ROC curves of energy detection scheme with different $\rho$

than $40 \%$. It means that cognitive users decide the spectrum is idle no matter whether there are primary users present. Consequently, cognitive users are harmful to licensed users when primary users are present. This situation often occurs in cognitive radio systems, particularly in lower signal-to-noise ratio environments.

This indicates that Energy detector is very sensitive to noise uncertainty. In order to guarantee a good performance, choosing a suitable threshold is very important. Traditional energy detection algorithms are based on a fixed threshold, and we have verified that the performance decreased under noise uncertainty environments. This indicates that the choice of a fixed threshold is no longer valid under noise uncertainty and threshold should be chosen flexible based on the necessary. In next section, energy detection algorithm with dynamic threshold is proposed.

\section{Dynamic Threshold Algorithm Description}

Since performance declined sharply as noise uncertainty, and cognitive users' accessing will be serious interference to licensed users, which should be avoided in dynamic spectrum access technology. For this reason, a new algorithm combatting the noise uncertainty is presented.

Assuming $\rho^{\prime}$ is the dynamic threshold factor and $\rho^{\prime}>1$ and $\rho^{\prime} \approx 1$, the value of dynamic threshold can be summarized in a single interval $\gamma^{\prime} \in\left[\gamma / \rho^{\prime}, \rho^{\prime} \gamma\right]$. In this section, we will consider the noise uncertainty and dynamic threshold respectively. From (8) and (9), it has:

$$
\begin{aligned}
P_{D} & =\min _{\gamma^{\prime} \in\left[\gamma / \rho^{\prime}, \rho^{\prime} \gamma\right]} \min _{\sigma^{2} \in\left[\sigma_{n}^{2} / \rho, \rho \sigma_{n}^{2}\right]} Q\left(\frac{\gamma^{\prime}-\left(P+\sigma^{2}\right)}{\sqrt{2 / N} P \sigma^{2}}\right) \\
& =Q\left(\frac{\gamma \rho^{\prime}-\left(P+\sigma_{n}^{2} / \rho\right)}{\sqrt{2 / N}\left(P+\sigma_{n}^{2} / \rho\right)}\right)
\end{aligned}
$$

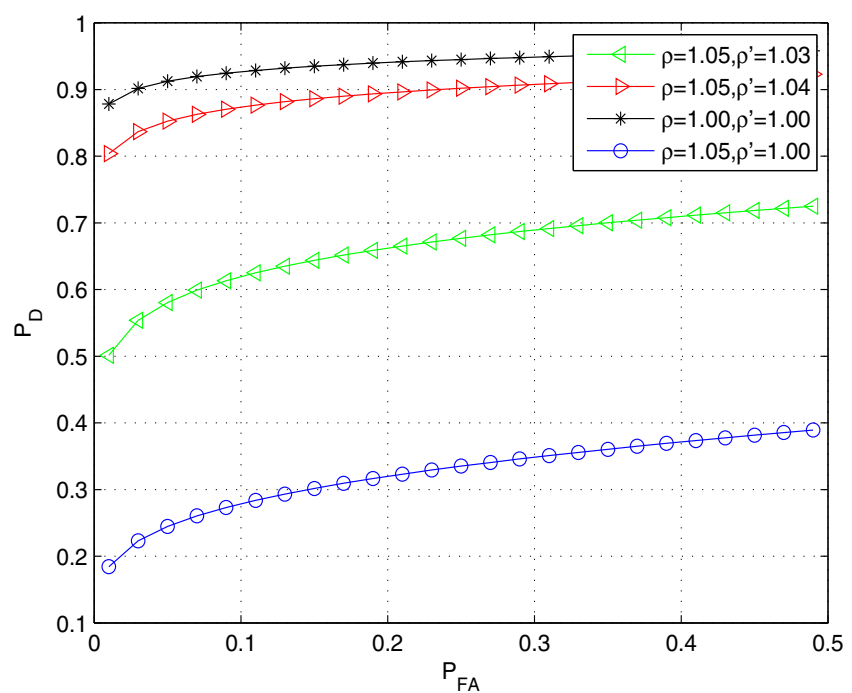

Fig. 3. ROC curves of energy detection scheme with different $\rho$ and $\rho^{\prime}$

$$
\begin{aligned}
P_{F A} & =\max _{\gamma^{\prime} \in\left[\gamma / \rho^{\prime}, \rho^{\prime} \gamma\right]} \max _{\sigma^{2} \in\left[\sigma_{n}^{2} / \rho, \rho \sigma_{n}^{2}\right]} Q\left(\frac{\gamma^{\prime}-\sigma^{2}}{\sqrt{2 / N} \sigma^{2}}\right) \\
& =Q\left(\frac{\rho^{\prime} \gamma-\rho \sigma_{n}^{2}}{\sqrt{2 / N} \rho \sigma_{n}^{2}}\right)
\end{aligned}
$$

Eliminating threshold $\gamma$ and getting inter-relationship of $P_{D}$, $P_{F A}, N, \rho, \rho^{\prime}$ and $S N R$

$$
\begin{aligned}
N & =2\left[\left(\rho / \rho^{\prime}\right) Q^{-1}\left(P_{F A}\right)-\rho^{\prime}(1 / \rho+S N R) Q^{-1}\left(P_{D}\right)\right]^{2} \\
& \times\left(\rho^{\prime} S N R+\rho^{\prime} / \rho-\rho / \rho^{\prime}\right)^{-2}
\end{aligned}
$$

In (13), when $\rho^{\prime} \approx \rho$ and $\rho^{\prime} / \rho \approx \rho / \rho^{\prime} \approx 1$, $\left(\rho^{\prime} S N R+\rho^{\prime} / \rho-\rho / \rho^{\prime}\right)^{-2} \approx(S N R)^{-2}$ and $\rho^{\prime}(1 / \rho+S N R) \approx(1+$ $S N R)$. we substitute (13) with the above approximate unequal expressions, and we can get that the numerical value of (13) is almost the same to (7). Therefore, dynamic threshold detection algorithm can overcome the noise uncertainty as long as a suitable dynamic threshold factor is chosen. Comparing (13) with (10), supposing $S N R=0.1$ and $\rho^{\prime}$ and $\rho$ both closer to 1 , it is clear that $\left(\rho^{\prime} S N R+\rho^{\prime} / \rho-\rho / \rho^{\prime}\right)^{-2} \gg(S N R-(\rho-1 / \rho))^{-2}$. Consequently, detection duration $N$ has been shortened largely with the same probability parameters $P_{D}$ and $P_{F A}$. It can be concluded that as long as the dynamic threshold factor is suitable, even if there is noise uncertainty, we can get a better spectrum performance. To attaining the same performance, the detection time of dynamic threshold energy detection algorithm is less than the traditional version.

Fig.3 is the numerical results of (7), (10) and (13). With the same parameters as before.

Where $\rho=1.00$ denotes that the average noise power keeps constant(without noise uncertainty); $\rho^{\prime}=1.00$ denotes that the algorithm did not use dynamic threshold(the threshold is 
fixed); otherwise, it represents cases with noise uncertainty and dynamic threshold. From Fig.3, it indicates that a tiny fluctuation of average noise power causes a sharp decline in detection performance. The dynamic threshold makes the performance improve significantly as the dynamic threshold factor increasing. If a suitable dynamic threshold factor is selected, the falling proportion of performance caused by noise uncertainty can be omitted and the performance may be more accurate.

\section{Conclusion}

Energy detection schemes based on fixed threshold are sensitive to noise uncertainty, a fractional fluctuate of average noise power will lead to the detection performance dropping quickly. According to its drawback, we present dynamic threshold spectrum detection algorithm in this paper. This algorithm has an accurate detection performance even if there is an serious noise uncertainty in the case of low signal-to-noise ratio. Theoretical analysis and numerical simulation results show that the proposed scheme improves the antagonism of noise uncertainty, and gains a good detection performance as long as a suitable dynamic threshold is chosen. In other words, the dynamic threshold energy detection algorithm can enhance the robust of combatting noise uncertainty and improve the veracity of spectrum sensing. How to acquire the practical detection threshold is our future work.

\section{ACKNOWLEDGMENT}

This paper is based upon work supported by National Natural Science Foundation (60872049 and 60871042), 863 Program of China under Grant (2007AA10Z235), and Teaching and Research Finances for the Returned Overseas Chinese Scholars.

\section{REFERENCES}

[1] M. McHenry, Spectrum white space measurements, June, 2003. Presented to New America Foundation Broadband Forum; Measurements by Shared Spectrum Company, http://www.newamerica.net/Download Docs/pdfs/Doc File 185 1.pdf.

[2] T.A. Weiss, F.Jondral, "Spectrum pooling: an innovative strategy for the enhancement of spectrum efficiency", IEEE Communications Magazine, Volume 42, Issue 3, Mar 2004. pp: 8 - 14.

[3] Ian F. Akyildiz, Won-Yeol Lee, Mehmet C. Vuran *, Shantidev Mohanty, "NeXt generation/ dynamic spectrum access/cognitive radio wireless networks: A survey", Computer Networks, Vol. 50, 2006, pp.21272159.

[4] Ian F. Akyildiz, Won-Yeol Lee, Mehmet C. Vuran, et al, "A Survey on Spectrum Management in Cognitive Radio Networks", IEEE Communications Magazine, Volume 46, Issue 4, April 2008. pp: 40-48.

[5] D.Cabric, S.M.Mishra, R.W.Brodersen, "Implementation issues in spectrum sensing for cognitive radios", IEEE Asilomar Conf.on Signals, Systems and Computers. Pacific Grove, USA, 2004. pp: 772-776.

[6] D.Cabric, A.Tkachenko, R.W.Brodersen, "Experimental study of spectrum sensing based on energy detection and netwotk cooperation", In ACM Int. Workshop on Technology and Policy for Accessing Spectrum. Boston, USA, 2006.

[7] A.Ghasemi, E.S.Sousa, "Collaborative spectrum sensing for opportunistic access in fading environments", In IEEE Int. Symposium on Dynamic Spectrum Access Networks. Baltimore, USA, 2005. pp: 131136.

[8] S.M. Mishra, A. Sahai, and R. W. Broderson, "Cooperative sensing among cognitive radios", In Proc. ICC 2005, Istanbul, Turkey, Jun.1115, 2006.
[9] Rahul Tandra, Anant Sahai, "SNR Walls for Signal Detection", EEE Journal of Selected Topics in Signal Processing, Vol.2, NO.1, Feb.2008. pp: 4-17.

[10] Rahul Tandra, "Fundamental limits on detection in low SNR", Master's thesis, University of California, Berkeley, 2003.

[11] S. M. Kay, "Fundamentals of Statistical Signal Processing: Detection Theory", Englewood Cliffs: Prentice-Hall, 1998, vol. 2. 